\title{
ANÁLISE DO DESEMPENHO DO CORPO DOCENTE DOS CURSOS DE ENGENHARIA CIVIL E PRODUÇÃO QUANTO A PESQUISA: O CASO DE UMA UNIVERSIDADE FORA DA SEDE
}

Natália Veloso Caldas de Vasconcelos - natalia.vasconcelos@ufersa.edu.br

Universidade Federal Rural do Semi-Árido

Rua Gamaliel Martins Bezerra,

59515-000 - Angicos - Rio Grande do Norte

Rafael de Azevedo Palhares - rafaelpalhareseng@ hotmail.com

Universidade Federal Rural do Semi-Árido

Rua Gamaliel Martins Bezerra,

59515-000 - Angicos - Rio Grande do Norte

Rayane Cabralda Silva - rayane_cabral@hotmail.com

Universidade Federal Rural do Semi-Árido

Rua Gamaliel Martins Bezerra,

59515-000 - Angicos - Rio Grande do Norte

Resumo: A atuação docente no ensino superior é composta por um tripé conhecido por todos que atuam na educação superior, o profissional deve exercer atividades de ensino, pesquisa e extensão. $O$ objetivo deste estudo é analisar o desempenho do corpo docente dos cursos de Engenharia de Produção e Engenharia Civil no tocante a pesquisa em uma Universidade fora da sede, para atingir este objetivo foi realizada a construção de um banco de dados considerando fontes de informações oficiais, institucionais, nacionais e pessoais para consolidar os dados e posteriormente analisa-los. O levantamento considerou docentes atuantes em cursos de Engenharia de Produção e Engenharia Civil, cursos com quantidade de docentes ativos (em atuação) semelhantes. Os resultados permitiram identificar que os docentes são atuantes em grupos de pesquisa, os docentes de Engenharia Civil produzem mais artigos para periódicos e os docentes de Produção destinam seus esforços para publicações em eventos, para realizar conclusões mais aprofundadas é necessário um estudo mais minucioso das informações.

Palavras-chave: Ensino Superior. Engenharia. Docentes. Pesquisa.

\section{INTRODUÇÃO}

Os procedimentos e competências relacionadas a construção, manufatura de materiais e fabricação de ferramentas são intrínsecos da Engenharia desde o início da civilização. Entretanto, ainda recente, apenas no século XVIII passou a associar-se ao conhecimento estruturado e organizado cientificamente. Desta forma, a evolução da Engenharia relaciona-se com os avanços tecnológicos e inovações científicas, tendo em vista que o autor Thomas Tregolf definiu em 1824 que compete a Engenharia o direcionamento de agentes da natureza para a melhor coabitação humana (OLIVEIRA et al., 2015). 
Adeodato et al. (2004) afirmam que a formação dos profissionais em engenharia, em muitos casos, preconiza e direciona o discente ao conhecimento em dados puramente concretos e sem reflexões. Desta forma, ainda para os autores, as Universidades geram profissionais restritos a ideias fixamente estabelecidas, sem concepções sociais e realísticas. Para Ferraz (1883) diante dos ensinamentos técnicos, o profissional em engenharia egressa da Universidade com limitações no que tange a situações reais, fazendo com que este profissional não esteja apto a lidar com experiências coletivas e nem com os fenômenos aleatórios intrínsecos da realidade. Assim, os autores concluem que o protagonismo das instituições de ensino como ambientes de formação deva concentrar-se em formar a sua comunidade acadêmica para a cidadania, isto é, educando-a para a pesquisa.

Demo (2001) destaca a importância da pesquisa na formação acadêmica, uma vez que a partir dela, o aluno passa a ter uma visão mais abrangente. Isto é, buscar fontes, maturidade para leitura e interpretação de dados, possibilitando a autonomia em descobrir uma diversidade de autores, teorias e ideologias, linhas de pensamento. Desta forma, além do conhecimento transmitido pelo docente em sala de aula, o discente adquire novas percepções além da visão do docente.

Ainda em relação às práticas de educação, é de suma importância considerar a existência do tripé composto pelo ensino, pesquisa e extensão. Grimoni e Garrossini (2013) consideram que a implementação do tripé de ensino de forma efetiva, exige que o docente adote uma compostura transformadora no âmbito da formação acadêmica. Isto é, faz-se evidente que o docente vá além do papel de instrutor e facilitador da informação e torne-se um mediador da construção, aplicação e divulgação do conhecimento.

Neste contexto, ainda de acordo com é Grimoni e Garrossini (2013), além de pensar na formação de inovadores profissionais da engenharia adequados as exigências do mercado de trabalho. Faz-se extremamente importante analisar a formação de um corpo docente ajustado à tais competências. Logo, esta pesquisa se propõe a analisar o desempenho do corpo docente dos cursos de Engenharia de Produção e Engenharia Civil no tocante a pesquisa em uma Universidade fora da sede.

Este estudo está estruturado em cinco seções: (I) Introdução, contemplando uma breve contextualização e revelando o objetivo da pesquisa. (II) Referencial Teórico, abordando o Papel do docente no ensino superior e a Avaliação da atuação docente (III) Procedimentos Metodológicos, contemplando as etapas da pesquisa (IV) Análise dos Resultados, na qual são discutidas as contribuições da pesquisa e por fim, (V) Considerações Finais.

\section{PAPEL DO DOCENTE NO ENSINO SUPERIOR}

Os cursos de graduação possuem a missão de preparar o discente para exercer uma profissão, assim, o processo de aprendizagem no ensino superior é longo e árduo, o qual dependerá das experiências, interesses e habilidades de cada acadêmico. (SBIZERA; DENDASCK, 2019), neste cenário destaca-se a figura do docente como mediador no processo de aprendizagem e formação do discente. De acordo com Pimenta e Lima (2010), este profissional deve possuir a capacidade de transformar a realidade social por meio da atividade docente. Benedito, Ferrer e Ferreres (1995) elencam algumas funções do docente de nível superior, entre elas: a pesquisa, à docência, a informação das suas investigações, a avaliação da docência, a interação com o mercado de trabalho, com a cultura, entre outras.

Segundo Silva, Dalmau e Amorim (2011) o docente de nível superior necessita identificar as necessidades do aluno e a partir disso, cabe realizar uma mediação, na qual aproxima o 
conteúdo ministrado à realidade do aluno para que o processo de ensino- aprendizagem ocorra de forma eficaz. Dessa forma, ensinar torna-se mais que lecionar uma disciplina, o profissional docente necessita de uma visão holística do processo educacional ao ponderar as necessidades do aluno, as competências da disciplina e as competências do curso.

Perrenoud (2000) elenca dez competências primordiais para desenvolver, de forma satisfatória, o papel de docente, são elas:

1. Preparar e administrar situações pedagógicas;

2. Conduzir a progressão da aprendizagem;

3. Idealizar e fazer evoluir os dispositivos de diferenciação;

4. Envolver os discentes em suas aprendizagens e em suas atividades;

5. Desenvolver trabalho em equipe;

6. Integrar a administração organizacional;

7. Apoiar e envolver;

8. Utilizar tecnologias atuais;

9. Enfrentar os problemas éticos da profissão;

10. Conduzir sua formação continuada.

Segundo o autor supracitado a construção de uma competência é compreendida como um artifício de aprendizagem, a qual se adquire na interação com o meio. Para Rios (2001) o caminho para a aquisição de uma competência é composto por tentativas e erros, momentos de hesitação, decisões contraditórias, inferências e raciocínios explícitos. O docente é exposto à ambientes complexos, vivos e multáveis, enfrentando dilemas individuais e grupais, onde o sucesso profissional está sujeito a sua capacidade de lidar com a complexidade de resolução de problemas práticos.

Vasconcelos (2002) pondera que um docente de ensino superior necessita possuir conhecimento técnico- científico para ter segurança do conteúdo ministrado; formação prática, ou seja, experiência profissional para a formação na área de formação dos alunos; formação política, isto é, ter consciência que a educação é isenta de neutralidade e formação pedagógica, a qual contribui para o desempenho metodológico e didático.

Mediante o exposto, nota-se que a docência superior é um caminho desafiador, exigindo do professor um conhecimento abrangente da sua área técnica e que possua senso crítico, além do conhecimento a respeito da realidade dos discentes. É necessário estar preparado o suficiente para ser capaz de produzir novos conhecimentos, inovando e criando (VASCONCELOS, 2002). O desenvolvimento das capacidades do docente relaciona-se diretamente com o desenvolvimento científico IES o qual o profissional está vinculado, assim faz-se necessário a avaliação do desempenho do docente para proporcionar reflexões críticas sobre a prática profissional.

\subsection{Avaliação da atuação do docente}

A avaliação da atuação docente nas IES é primordial para o desenvolvimento profissional dos docentes, assim como para o crescimento institucional. O processo de avaliação visa embasar a avaliação de estágios probatórios, regimes de trabalho e o progresso individual na carreira docente. Vale destacar que o desenvolvimento do professor de nível superior é considerado um indicador importante para a boa avaliação da instituição, ou seja, o desenvolvimento satisfatório das atividades docentes reflete na qualidade do ensino ofertado nas IES (PINTO; OLIVEIRA, 2005).

A avaliação da atuação docente nas IES é primordial para o desenvolvimento profissional dos docentes, assim como para o crescimento institucional. O processo de avaliação visa 
embasar a avaliação de estágios probatórios, regimes de trabalho e o progresso individual na carreira docente. Vale destacar que o desenvolvimento do professor de nível superior é considerado um indicador importante para a boa avaliação da instituição, ou seja, o desenvolvimento satisfatório das atividades docentes reflete na qualidade do ensino ofertado nas IES (PINTO; OLIVEIRA, 2005). Os docentes de nível superior podem ser avaliados pela eficiência e eficácia do desenvolvimento das atividades de Ensino- Pesquisa e Extensão.

Parrenoud (2000) afirma que a inserção dos discentes em projetos de Ensino- Pesquisa e Extensão proporciona o desenvolvimento de competências que são impossíveis de serem adquiridas em uma sala de aula tradicional, essas atividades desenvolve as múltiplas inteligências que o aluno necessita para a sua formação profissional. Magalhães (2004) cita habilidade de criticar, desenvolver trabalho em equipe, planejar, sugerir e realizar ações como competências desenvolvidas na Pesquisa- Ensino e Extensão. Mediante o exposto percebe-se a o envolvimento dos docentes no tripé da universidade impacta diretamente na qualidade da formação profissional do aluno e por consequência na avaliação da IES.

Para Rios (2004) os resultados das avaliações do docente apresentam uma oportunidade de crescimento e desenvolvimento professor e instigam a reflexão, além de proporcionar a implementação de novas práticas pedagógicas no ensino superior. Destaca-se que as avaliações não são somente uma medida de desempenho e sim um processo de controle da instituição que corrobora para a aplicação planejada de ações preditivas e corretivas.

Para Rios (2004) os resultados das avaliações do docente apresentam uma oportunidade de crescimento e desenvolvimento professor e instigam a reflexão, além de proporcionar a implementação de novas práticas pedagógicas no ensino superior. Destaca-se que as avaliações não são somente uma medida de desempenho e sim um processo de controle da instituição que corrobora para a aplicação planejada de ações preditivas e corretivas.

\section{PROCEDIMENTOS METODOLÓGICOS}

O Campus objeto de estudo desta pesquisa é decorrente do Programa de Reestruturação e Expansão das Instituições Federais de Ensino Superior (REUNI), que possibilitou a sua criação em dezembro de 2008 e início de suas atividades acadêmicas em março de 2009. Atualmente, o referido Campus oferta sete cursos de graduação, são eles: Sistema de Informação, Bacharelado em Ciência e Tecnologia, Bacharelado em Sistemas de Informação, Licenciatura em Computação e Informática, Pedagogia, Engenharia Civil e, Engenharia de Produção.

A presente pesquisa foi realizada em seis principais etapas conforme apresentado na Figura 1. Neste sentido, a primeira etapa para o desenvolvimento do trabalho compreende a delimitação do escopo do estudo. Assim, optou-se pela análise dos cursos de Engenharia Civil e Engenharia de Produção, visto serem os dois cursos de Engenharia ofertados pelo Campus. Vale salientar que o curso de Engenharia Civil teve sua primeira entrada de discentes em 2013, sendo o primeiro curso de engenharia do campus e o curso de Engenharia de Produção teve suas atividades iniciadas no ano de 2014. A quantidade de docentes que compõe os dois cursos totaliza 25, enfatizando que alguns desses docentes ministram disciplinas nos dois cursos, em áreas que são compartilhadas como por exemplo: meio ambiente e segurança do trabalho.

Figura 1 - Etapas da Pesquisa 


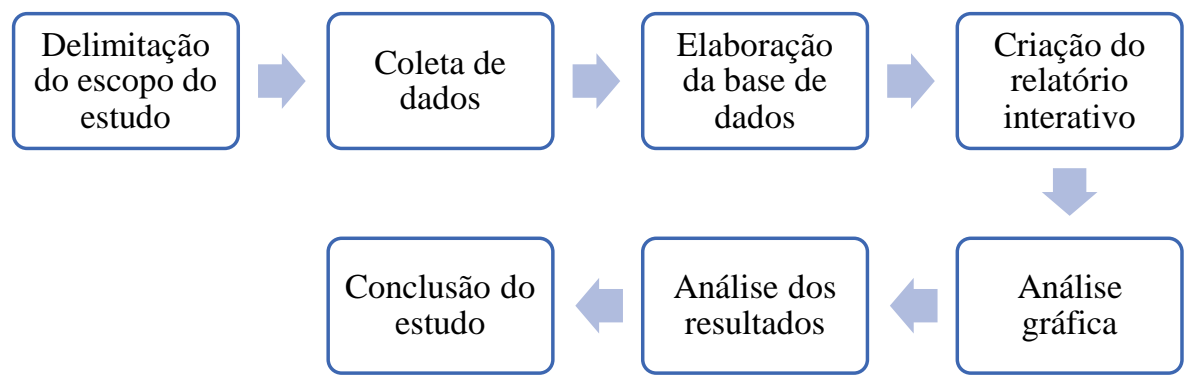

Fonte: Esta Pesquisa (2020)

Em seguida, no período entre 10/06/2020 a 05/07/2020 fez-se a coleta de dados no Sistema Integrado de Gestão de Atividades Acadêmicas (SIGAA) público da instituição, na Gestão de Serviços de Tecnologias da informação da Superintendência de Tecnologia da Informação e Comunicação (GLPI-SUTIC), Diretório dos grupos de pesquisa e a Plataforma Lattes, as duas últimas associam-se ao Conselho Nacional de Desenvolvimento Científico e Tecnológico (CNPq). De posse dos dados, elaborou-se uma base de dados contemplando o quantitativo em relação a participação dos docentes em grupos e projetos de pesquisa, coordenação de projeto de pesquisa e número de publicações em periódicos bem como em eventos científicos no Triênio (2017-2019).

Posteriormente, auxiliado pela ferramenta Google Data Studio, foi possível construir o relatório interativo e condensado das informações, apoiando nas seguintes etapas de análise gráfica, análise dos resultados e conclusão do estudo.

\section{RESULTADOS E DISCUSSÕES}

Nesta seção do presente estudo vamos analisar os resultados obtidos através da coleta de dados conforme descrito na metodologia.

A primeira análise trata-se da caracterização do grupo dos docentes no tocante a titulação e status do docente na instituição, visto que alguns docentes do quadro efetivo podem estar afastados para qualificação, o Gráfico 1 mostra os dados.

Gráfico 2 - Caracterização do Grupo de Docentes

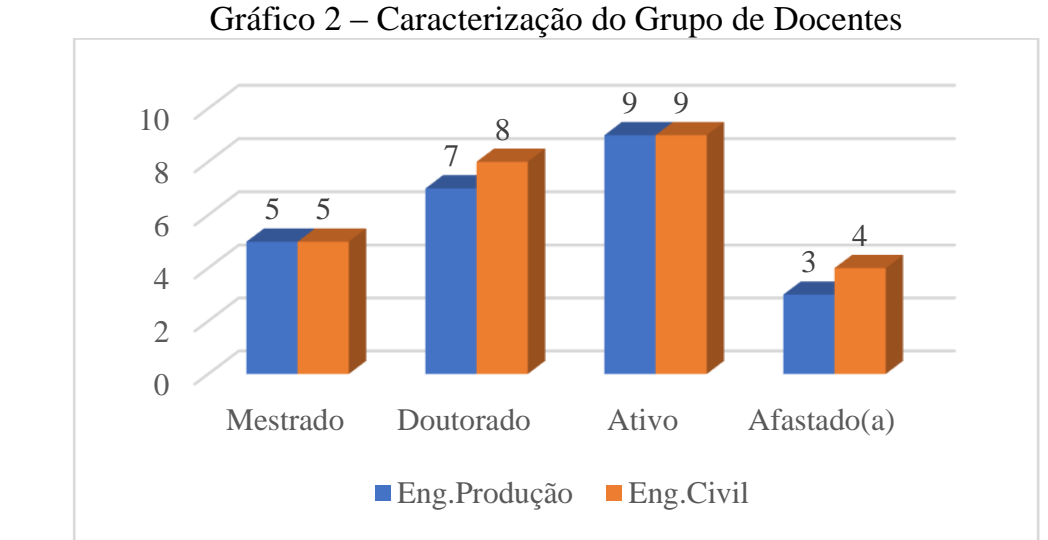

Fonte: Esta Pesquisa (2020)

O grupo de docentes de Engenharia Civil, 13 docentes, é ligeiramente maior que o de Produção, que conta com 12 docentes. Há um equilíbrio entre as titulações e também ambos apresentam a mesma quantidade de docentes ativos, ou seja, atuando na instituição. 
No tocante a participação em grupos de pesquisa, de acordo com o Gráfico 2, há uma leve participação maior dos docentes de Civil. Enquanto os docentes de Engenharia de Produção estão envolvidos em 14 grupos de pesquisa, os docentes de Civil colaboram em 19 grupos. É importante frisar que alguns docentes participam de mais de um grupo de pesquisa, como exemplo um docente de Civil que está listado em 4 grupos de pesquisa.

Gráfico 2 - Participação em Grupo de Pesquisa



Fonte: Esta Pesquisa (2020)

Uma questão que é relevante apresentar é a participação como líder em grupos de pesquisa, cada curso apresentou 4 docentes caracterizados como líderes de grupos. Outro indicador interessante é que $80 \%$ dos docentes analisados participam de pelo menos um grupo de pesquisa, o que indica um envolvimento substancia dos docentes em grupos. Porém, se o trabalho desenvolvido nos grupos é convertido em resultados, através de publicações, será analisado adiante.

A análise realizada em sequência considerou os projetos de pesquisa desenvolvidos no âmbito da universidade, devidamente cadastrados no sistema institucional. O Gráfico 3 mostra os resultados, no qual o corpo docente de Civil se apresentou mais atuantes em todos os anos da análise. É possível notar um desenvolvimento mis substancial de projetos de pesquisa no curso de Engenharia de Produção no ano de 2019, este fato pode ter ocorrido devido a nos anos anteriores (2017-2018) o corpo docente de Engenharia de Produção não contava com uma quantidade expressiva de docentes doutores, e como a titulação é fator relevante na obtenção de bolsas de pesquisa, pode ter impactado no indicador.

Gráfico 3 - Desenvolvimento de Projetos de Pesquisa

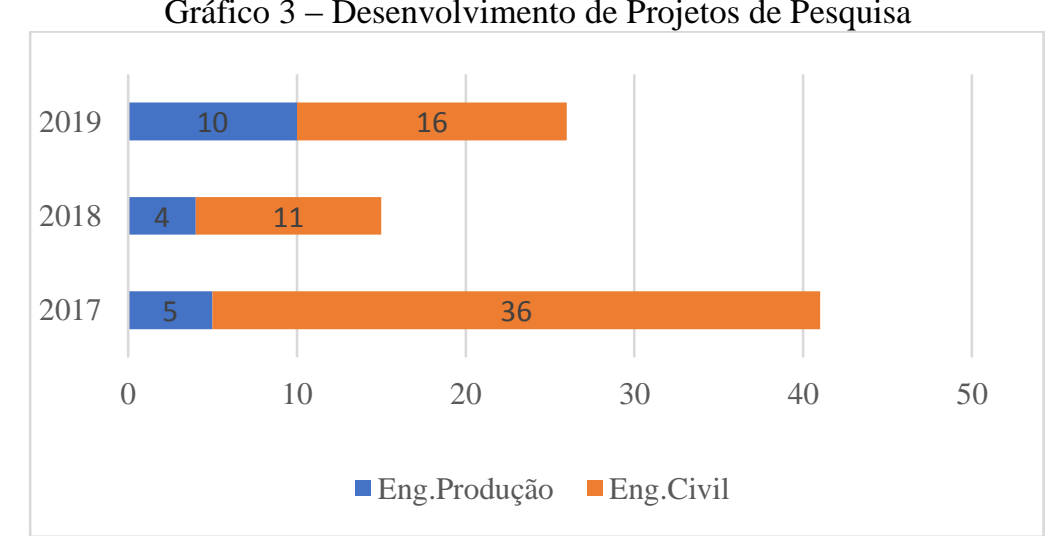

Fonte: Esta Pesquisa (2020) 
O gráfico 4 apresenta o volume de publicações em periódicos. O volume de publicação é crescente, no grupo de docentes de Engenharia. Quando é analisado os cursos, se nota um salto entre 2017 e 2018 no curso de Civil, já no curso de Produção o salto ocorre entre 2018 e 2019, intervalo que também se identifica uma aproximação no número de publicações entre os cursos.

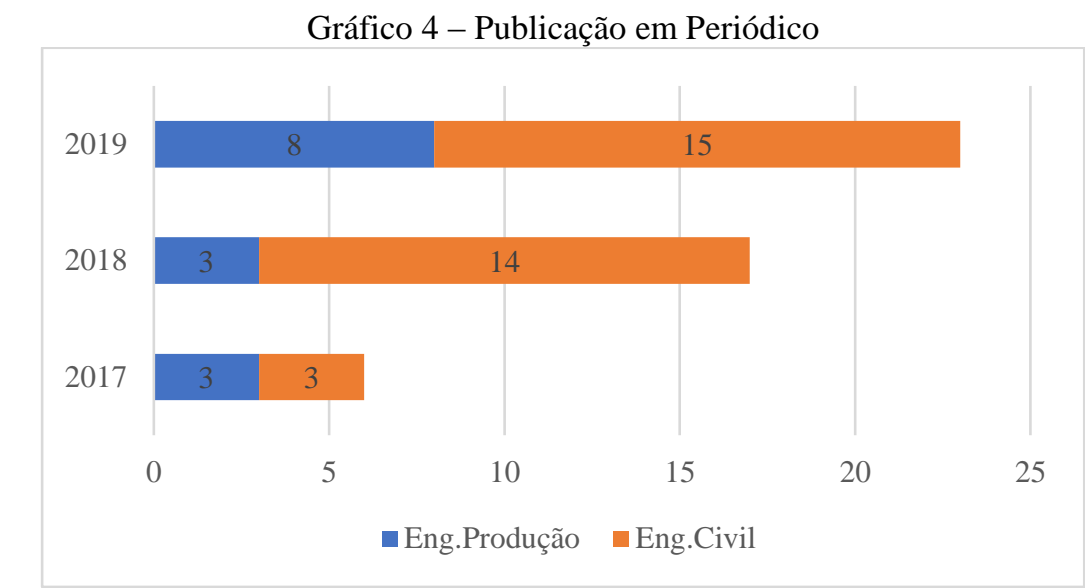

Fonte: Esta Pesquisa (2020)

Para concluir as análises gráficas, o Gráfico 5 trata das publicações em eventos. Enfatizase que não houve diferenciação entre eventos internacionais, nacionais ou regionais, foram consideradas as publicações em anais de eventos. Há uma superioridade no volume de artigos publicados em eventos pelos docentes de Engenharia de Produção, diferença idêntica de 12 publicações em 2017 e 2019, porém em 2018 o número expressivo de publicações, atingindo o volume máximo de 98 publicações em 2018, considerando as publicações dos docentes dos dois cursos.



Fonte: Esta Pesquisa (2020)

Para finalizar as análises no tocante as atividades de pesquisa dos docentes dos cursos de Engenharia Civil de Produção, foi realizada uma análise conjunta, considerando o grupo de professores que atuam nos dois cursos, para realizar um diagnóstico sobre a atuação em projeto de pesquisa, publicação em periódicos e publicação em eventos no triênio já mencionado. A Tabela 1, apresenta o volume total de projetos e publicações e também uma média por docente a cada ano. 
Tabela 1 - Painel de Projeto, Publicação em Periódico e Eventos.

\begin{tabular}{l|r|r|r}
\hline Indicadores & $\mathbf{2 0 1 7}$ & $\mathbf{2 0 1 8}$ & $\mathbf{2 0 1 9}$ \\
\hline Total de projetos & 41 & 15 & 26 \\
\hline Média & $\mathbf{1 , 6 4}$ & $\mathbf{0 , 6}$ & $\mathbf{1 , 0 4}$ \\
\hline Publicações em Periódicos & 6 & 17 & 23 \\
\hline Média & $\mathbf{0 , 2 4}$ & $\mathbf{0 , 6 8}$ & $\mathbf{0 , 9 2}$ \\
\hline Publicações em Eventos & 50 & 98 & 56 \\
\hline Média & $\mathbf{2}$ & $\mathbf{3 , 9 2}$ & $\mathbf{2 , 2 4}$ \\
\hline
\end{tabular}

Fonte: Esta Pesquisa (2020)

A Tabela 1 apresenta alguns resultados alarmantes quando se analisa a média de projetos, que apresentou uma queda em 2018, e voltou a subir de maneira sutil em 2019, porém um indicador que pode ser melhorado, quando se parte do pressuposto que as pesquisas desenvolvidas nas universidades são resultados de projetos, sendo assim haver um volume grande projetos de pesquisa na universidade é algo positivo para docentes e discentes.

Quando se analisa o número de publicações em periódico do grupo de docentes por ano, as médias mostram que o envolvimento em grupos de pesquisa não se traduz em resultados de maneira efetiva, visto que o número de publicações por docente é baixo, não chegando nem a 1 publicação por docente, a cada ano. $\mathrm{O}$ indicador é preocupante principalmente pelo grupo envolver uma grande quantidade de docentes doutores, o que deveria representar um número maior de publicações em periódicos, porém outro ponto que deve ser evidenciado é que a média está apresentando um leve crescimento.

Os valores de publicações em eventos são um pouco mais altos, considerando também o nível de complexidade da publicação, e também o envolvimento dos discente para a publicação desses artigos destinados aos eventos, que permitem a participação discente o que gera uma motivação maior para publicações.

\section{CONSIDERAÇÕES FINAIS}

O presente estudo foi proposto para analisar o desempenho do corpo docente dos cursos de Engenharia de Produção e Engenharia Civil no tocante a pesquisa em uma Universidade fora da sede, o mesmo foi atingido quando apresentadas as informações acerca de: participação em grupos de pesquisa, se o docente era líder ou não de grupos, realização de projetos de pesquisa, publicação em período e publicações em eventos, os dados analisados levaram em consideração os projetos e publicações desenvolvidos entre 2017 e 2019. As análises realizadas de modo comparativo entre os cursos evidenciaram: uma maior atuação dos docentes de Engenharia Civil em projetos de pesquisa e em publicações em periódicos, em contrapartida os docentes de Engenharia de Produção se destacaram por suas publicações em eventos.

Porém de modo geral, os indicadores mostram que o envolvimento com atividade de pesquisa ainda está aquém, quando se analisa que grande parte do corpo docente dos cursos obtém a titulação de doutorado.

Essa análise é de extrema relevância para auxiliar a gestão das instituições de ensino superior no tocante ao desempenho dos docentes em suas atividades, que são basicamente: ensino, pesquisa e extensão.

Como recomendações para trabalhos futuros a continuidade do levantamento é extremamente importante para se avaliar a evolução das pesquisas e do envolvimento dos 
docentes. Além de ser necessário realizar uma análise mais aprofundada considerando também a extensão e as atividades de ensino, visto que cada docente pode haver preferência para uma vertente do tripé.

\section{REFERÊNCIAS}

ADEODATO, Marise Tissyana et al. O ensino da sustentabilidade em cursos de graduação em engenharia civil integrando pesquisa e extensão: a experiência da UFSCar. In: Congresso Brasileiro de Educação em Engenharia, 10., 2004, Brasilia. Anais [...]. Brasilia, 2004.

BENEDITO, A. V.; FERRER. V.; FERRERES, V.; La formación universitária a debate. Barcelona: Publicaciones Universitat de Barcelona, 1995.

DEMO, Pedro. Universidade e reconstrução do conhecimento. In: Universidade Federal de São Carlos - Pró-Reitoria de Graduação. Ensino de Graduação: Reflexões e Proposições n³: Diretrizes curriculares e projeto pedagógico. UFSCar: São Carlos, mar. 2001. pp.75-96.

GRIMONI, Jose Aquiles Baesso; GARROSSINI, Daniela Fávaro. Capacitação e formação continuada para docência em cursos superiores de tecnologia e de engenharia. In: congresso brasileiro de educação em engenharia, 41., 2013, Gramado - Rs. Proposta de Sessão Dirigida. Gramado - Rs: ABENGE, 2013.

MAGAlHÃES, H. G. D. A pedagogia do êxito: projetos de resultado. Petrópolis: Vozes, 2004.

OLIVEIRA, Vanderli Fava de et al. A expansão do número de cursos e de modalidades de engenharia. In: Congresso Brasileiro De Educação Em Engenharia, 42., 2015, Mauá. Anais [...] . Mauá: Abenge, 2015. p. 1-10.

PERRENOUD, P. Dez novas competências para ensinar. Trad. Patrícia Chitoni Ramos. Porto Alegre: Artes Médicas Sul. 2000.

PIMENTA, S. G.; LIMA, M. S. L. Estágio e docência. 7. ed. São Paulo: Cortez, 2010. RIOS T. A. Compreender e ensinar: por uma docência de melhor qualidade. 2.ed. São Paulo: Cortez, 2001.

RIOS, M. P. G. Meta-avaliação de docentes no ensino superior. Tese (Doutorado) Pontifícia Universidade Católica de São Paulo, São Paulo, 2004.

SBIZERA, C. L. G. A.; DENDASCK, C. V. Processo ensino/aprendizagem na universidade. Revista Científica Multidisciplinar Núcleo do Conhecimento. Ano 04, $5^{\text {a }}$ ed., vol. 03, p. 15-26, 2019. ISSN: 2448-0959

SILVA, A. L. G. da; DALMAU, M. B. L.; AMORIM, S. S. de. Competência docente no ensino superior: especificidades requeridas na educação à distância. In: XI Congresso Internacional sobre Gestão Universitária na América do Sul, 2011, Florianópolis. Anais. Florianópolis, 2011. 
VASCONCELOS, M. L. M. C. Contribuindo para a formação de professores universitários: relato de experiências. In: MASETTO, M. (Org). Docência na Universidade. 4. ed. Campinas: Papirus, 2002.

\title{
ANALYSIS OF THE TEACHER'S PERFORMANCE OF THE CIVIL ENGINEERING AND PRODUCTION COURSES IN RESEARCH: THE CASE OF A UNIVERSITY OUTSIDE THE HEADQUARTERS
}

\begin{abstract}
The teaching performance in higher education is composed of a tripod known to all who work in higher education, the professional must carry out teaching, research and extension activities. The objective of this study is to analyze the performance of the teaching staff of the Production Engineering and Civil Engineering courses with regard to research at a University outside the headquarters, in order to achieve this objective, a database was built considering official information sources, institutional, national and personal data to consolidate the data and then analyze it. The survey considered professors working in Production Engineering and Civil Engineering courses, courses with a similar number of active (working) teachers. The results allowed to identify that the professors are active in research groups, the Civil Engineering professors produce more articles for journals and the Production professors dedicate their efforts to publications in events, to make more in-depth conclusions, a more detailed study of the information is necessary.
\end{abstract}

Keywords: Higher education. Engineering. Teachers. Research. 